\title{
ENVEJECIMIENTO, MASA MUSCULAR Y ENTRENAMIENTO DE LA FUERZA: UNA REVISIÓN
}

\author{
AGING, MUSCLE MASS AND STRENGTH TRAINING: A REVIEW
}

\author{
Santiago Adolfo Arboleda Franco* \\ Fredy Alonso Patiño Villada** \\ José Antonio de Paz Fernández***
}

\section{Resumen}

\begin{abstract}
En este artículo se revisan los fundamentos sobre los cuales se ha reconocido la fuerza como elemento esencial en las prescripciones de ejercicio físico para la salud, específicamente en las personas mayores. Se describen las implicaciones de la sarcopenia como síndrome propio de este periodo vital y los hallazgos sobre la importancia de la masa muscular en el equilibrio homeostático mediante su función endocrina. También se examina lo propuesto desde consensos y declaraciones de asociaciones científicas sobre el entrenamiento de distintas manifestaciones de la fuerza en un contexto de salud y se revisa lo hallado en algunos meta-análisis y revisiones sistemáticas recientes sobre los efectos de este entrenamiento en distintas variables relacionadas con la capacidad funcional. Por último, se señalan algunos horizontes de investigación en el tema.
\end{abstract}

Palabras clave: ancianos, sarcopenia, capacidad funcional, aptitud muscular.

\section{Abstract}

This paper reviews the foundations in which the strength has been recognized as an essential element in the requirements of physical exercise for health, especially in the elderly. The implications of sarcopenia are described as proper syndrome during this vital period and the findings about the importance of muscle mass in the homeostatic balance through its endocrine function. It also scans what it is proposed from consensus and statements from scientific associations, about different strength training manifestations in a health context and it reviews the findings in some meta-analysis and variables related to the functional capacity. Finally, it makes an approach in some research perspectives regarding this issue.

Keywords: elderly, sarcopenia, functional capacity, muscle aptitude.

Fecha de recepción: 12 de diciembre de 2013

Fecha de aprobación: 28 de marzo de 2014

\footnotetext{
Doctorando en Ciencias de la Actividad Física y el Deporte, Universidad de León, España. Profesor Asistente Área Educación Física y Deporte, Universidad del Valle, Colombia. Correos electrónicos: santiago.arboleda@correounivalle.edu.co - cibertiago@yahoo.com

** Doctorando en Ciencias de la Actividad Física y el Deporte, Universidad de León, España. Profesor Asistente Instituto Univer sitario de Educación Física, Universidad de Antioquia, Colombia. Correo electrónico: fredpa18@hotmail.com

*** Doctor en Medicina y Cirugía. Especialista en Medicina Deportiva. Profesor Titular Departamento de Fisiología. Universidad de León, España. Correo electrónico: japazf@unileon.es
} 


\section{INTRODUCCIÓN}

En la década de los noventa, aumentó la evidencia científica que relacionó la baja condición musculoesquelética con mayor riesgo para la salud, y en lo referente al envejecimiento, Rosemberg propuso el concepto sarcopenia (de las expresiones griegas sarx: carne y penia: pérdida) para referirse a la pérdida de masa muscular relacionada con la edad (1997); esta, a su vez se reconoció como factor de riesgo para la pérdida de autonomía y funcionalidad y como causa desencadenante de discapacidad (Rosemberg, 1997; Cruz-Jentof et al., 2010; Barbat-Artigas et al., 2012). Por lo mismo, se recomendó el entrenamiento de fuerza como parte integral del acondicionamiento físico en adultos sanos, sugiriéndose una intensidad suficiente para mejorar la fuerza y la masa libre de grasa (Kraemer et al., 2002). También se desmitificó este tipo de entrenamiento en personas mayores (Frontera et al., 1988; Fiaratone et al., 1990).

Es más reciente la comprensión del papel desempeñado por el músculo en la regulación de la homeostasis y su relación con varias enfermedades (Pedersen y Febbraio, 2008; Pedersen, 2011), así como la precisión del concepto sarcopenia, hoy admitido como síndrome y con criterios unificados para su diagnóstico, al adicionarse a la baja masa muscular, la valoración de su estado de funcionalidad, expresado en la fuerza o la performance motriz. Con esto se configuraron tres estadios de diagnóstico: presarcopenia, sarcopenia y sarcopenia severa, con base en un algoritmo que valora el estado de la relación entre masa y función muscular según el tipo de pruebas utilizadas (Cruz-Jentof, et al., 2010).

Actualmente, es indiscutible que un pobre fitness muscular se relaciona con mayor morbilidad y mortalidad por todas las causas y obtenerlo o preservarlo se asocia a buena postura y reducción de problemas de espalda, bienestar y calidad de vida, reducción del riego de osteoporosis, control de peso y un efecto preventivo frente a enfermedades crónicas y metabólicas (FitzGerald et al., 2004). Existen consensos y directrices que definen de modo claro los fundamentos del entrenamiento de fuerza en el contexto de la salud (Kraemer et al., 2002; Nelson et al., 2007; Garber et al., 2011).No obstante, en países en vía de desarrollo el papel desempeñado por los profesionales de la educación física y el deporte en el tema específico de la prescripción del ejercicio para la salud aún debe fortalecerse, por lo que resulta necesario replicar esta información; para el caso, atendiendo a los enfoques actuales sobre el entrenamiento de la fuerza para las personas mayores, mediante la revisión de algunos antecedentes y estudios actuales, principalmente meta-análisis, revisiones sistemáticas y pronunciamientos de asociaciones científicas, localizados en las bases de datos PubMed-MEDLINE, ISI Web Knowledge y Scopus. Con todo lo anterior, esta revisión tiene como objetivo examinar la relación entre el envejecimiento, la masa muscular y las características y efectos del entrenamiento de fuerza con fines de salud en las personas mayores e identificar algunos temas pendientes de investigación.

\section{ENVEJECIMIENTO Y SARCOPENIA}

La sarcopenia se atribuye a la interacción de múltiples factores, pero el primero en mediar es el envejecimiento. Se ha establecido que la masa muscular se empieza a perder aproximadamente desde los 45 años a razón de 1,9 y 1,1 kilogramos por década, en hombres y mujeres respectivamente; dicha pérdida afecta más a los miembros inferiores y tiende a ser progresiva con la edad (Jansen et al., 2000). En un estudio de Frontera et al. (2000), luego de 12 años de seguimiento a un grupo de hombres aparentemente sanos de 65,4 años, se encontró disminución de un $29 \%$ en la fuerza medida isocinéticamente y del 14,7\% en el área de sección trasversal (CSA por sus siglas en inglés) de la musculatura de miembros inferiores, también un menor número de capilares por fibra. No obstante, los autores sugirieron que la variabilidad de los cambios entre individuos, en cuanto a tamaño de músculo y tipo de fibra, es amplia, y estas reducciones pueden ser más agudas en sujetos desacondicionados o enfermos.

La disminución de masa muscular y fuerza, concomitante con el envejecimiento, también se ha explicado desde las reducciones en la función de los sistemas somatosensorial y neuromotor, relacionadas con la atrofia selectiva de las fibras tipo IIx, por apoptosis de motoneuronas (Deschenes, 2004; Trappe, 2009). También por los cambios en la regulación hormonal (Szulc, et al., 2004), la disminución en el consumo calórico y proteico y el aumento en la actividad de los mediadores inflamatorios (Deschenes, 2004; Raj et al., 2010). A esto se suman los cambios en la arquitectura muscular relacionados con alteraciones en la longitud y el ángulo de penación de los fascículos, los cuales se reducen con 
la edad (Raj et al., 2010). Así, el descenso en el nivel de actividad física cumple un doble rol, siendo a la vez causa y consecuencia de este síndrome.

Los efectos de la sarcopenia afectan todas las manifestaciones de la fuerza, principalmente en su componente concéntrico (Frontera et al., 2000; Deschenes, 2004; Cruz-Jentof et al., 2010), reflejándose principalmente en la disminución de la potencia, la velocidad de la marcha, el incremento en el riesgo de caídas y la reducción en la capacidad para desarrollar actividades de la vida diaria (AVD) (Deschenes, 2004; Raj et al., 2010; Cruz-Jentof et al., 2010), lo cual también tiene implicaciones en la funcionalidad de las personas mayores, especialmente para su calidad de vida al aumentar el riesgo de discapacidad y dependencia (Jansen et al., 2002; Cruz-Jentof et al., 2010). Todo esto es factor de preocupación de los sistemas sanitarios de los países por el alto costo económico y social que se genera.

Otro concepto importante para la valoración de la función muscular, y relacionado con la sarcopenia, es el de calidad muscular, que establece la relación entre el músculo y la fuerza por unidad de masa muscular. Esta relación se puede hacer con la fuerza máxima o la potencia y en términos de salud es útil para la valoración del riesgo de discapacidad, aunque todavía no hay consenso en un método estandarizado y puntos de corte que determinen ese riesgo (Barbat et al., 2012).

\section{IMPORTANCIA DE LA MASA MUSCULAR EN EL CONTEXTO DE LA SALUD}

La perspectiva que ligaba la función muscular principalmente a la contracción y la producción de movimiento se ha ampliado recientemente para indagar por otras propiedades e implicaciones de este tejido en la homeostasis (Pedersen, 2011). Sobre esto, en principio solo se reconocía el rol que el músculo ejercía en la regulación del metabolismo energético a través de la oxidación de la glucosa y los lípidos, sin embargo, estudios recientes han confluido en atribuir una función endocrina y paracrina al tejido muscular, por cuanto se ha encontrado que produce proteínas denominadas miokinas (Interleucinas 6, 8 y 15 entre muchas otras), cuya función es de tipo hormonal. (Febbraio y Pedersen, 2005; Pedersen y Febbraio, 2008). La secreción de miokinas está ligada a la contracción muscular, por lo que el ejercicio es un factor potenciador de su produc- ción y estas, a su vez, también guardan relación con el sistema inmunitario, por cuanto regulan a las adipokinas (proteína estimuladora de acilación -ASP, TNF$\alpha$, IL-6, Resistina) en procesos pro y antiinflamatorios (Petersen y Pedersen, 2005).

Se ha establecido que la inflamación crónica es responsable de problemas para la salud como la resistencia a la insulina, arterioesclerosis, neurodegeneración, formación de tumores y todo lo que esto conlleva (Pedersen et al., 2000; Brandt y Pedersen, 2010) y en cierto modo la acumulación excesiva de grasa visceral juega un papel importante, por cuanto desde allí se producen adipokinas proinflamatorias. Por lo mismo, el ejercicio físico ejerce un efecto antiinflamatorio y otros específicos sobre la grasa visceral, atribuible en buena medida a la acción de las miokinas. Estas trabajan localmente en el músculo y ejercen efectos sobre vías de señalización involucradas en la oxidación de las grasas y el consumo de la glucosa, además de la modulación de procesos inflamatorios antes mencionada (Febbraio y Pedersen, 2005; Petersen y Pedersen, 2005).

La IL-6 es una de las miokinas más estudiadas (Febbraio y Pedersen, 2005; Pedersen y Febbraio 2008), es producida tanto en el tejido muscular como graso y dependiendo del proceso metabólico, puede asumir funciones pro o antiinflamatorias. La isoforma secretada por el tejido muscular es antiinflamatoria, incluso estimula la producción de otras citokinas con función similar como las IL-1ra y la IL-10; además regula la inflamación aguda e inhibe los niveles circulantes de TNF- $\alpha$ contrarrestando la resistencia a la insulina (Brandt y Pedersen, 2010). Varios estudios han comprobado su sensibilidad al ejercicio (Febbraio y Pedersen, 2005; Pedersen 2011). También se ha dicho que otras interleukinas como la IL-8 y la IL- 15 son especialmente sensibles al entrenamiento de fuerza (Pedersen y Febbraio, 2008). Además de las miokinas mencionadas, otras como el Factor Neurotrópico Derivado del Cerebro (BDNF), el Factor de Crecimiento Fibroblástico 21 (FGF-21) y la Visfatina, también cumplen funciones que favorecen la respuesta inmunitaria (Pedersen, 2011).

Sintetizando lo anterior, el envejecimiento en sí mismo predispone a cierto estado de inflamación, y si se combina con el sedentarismo y la obesidad se aumentan las adipokinas proinflamatorias, lo que, ligado a la dismi- 
nución en la producción de miokinas, desencadena en muchos de los problemas de salud que afectan en esta etapa de la vida, como diabetes, arterioesclerosis, síndrome metabólico, cáncer entre otros (Pedersen, et al., 2000). De allí la importancia del rol que juega el ejercicio y, dentro de este, el que desempeña el tejido muscular como regulador endocrino e inmunitario (Febbraio y Pedersen, 2005; Pedersen, 2011).

\section{CARACTERÍSTICAS DEL ENTRENAMIENTO DE FUERZA EN PERSONAS MAYORES}

El interés en el estudio del entrenamiento de la fuerza en personas mayores se dio desde la década de los noventa, cuando las primeras investigaciones indagaron por las características de la evolución de esta capacidad y el comportamiento de sus distintas manifestaciones en dependencia de la edad y el sexo (Marques et al., 2013). Dos referentes importantes son los estudios de Frontera et al. (1988) y Fiaratone et al. (1990). En el primero se realizó un entrenamiento de tres meses, tres veces por semana, al 80\% de 1RM, en hombres entre 60-72 años, que produjo mejoras del 8,5 y $18,5 \%$ en la fuerza isocinética de los flexores y extensores de rodilla respectivamente, e incrementos de más del 100\% para la RM. Uno de los hallazgos más importantes para la época, fue la hipertrofia del cuádriceps femoral, encontrándose incrementos significativos, tanto del CSA del cuádriceps, como en el área de las fibras musculares obtenidas por biopsia (Frontera, et al., 1988). Por su parte, Fiaratone et al. (1990) entrenaron ancianos frágiles mayores de 90 años tres veces por semana durante dos meses, mediante extensiones del cuádriceps, encontrando incrementos clínicamente significativos en CSA del $10,9 \%$, mejoras en la movilidad funcional y ganancias en la fuerza superiores al $170 \%$.

Numerosos estudios han abordado el tema y hay disponible directrices y consensos de expertos, fundamentados en evidencia tipo A (Kraemer et al., 2002; Nelson et al., 2007; Chodzko-Zajko et al., 2009; Peterson et al., 2010; Ratamess et al., 2011; Garber et al., 2011). Así, en las recomendaciones de la American Hearth Association (Nelson, et al., 2007) y su posterior actualización por Chodzko-Zajko et al. (2009) como pauta del American College of Sports Medicine (ACSM) para personas mayores, o en las directrices generales de 2011 de la misma institución (Garber et al., 2011), donde se establecen criterios claros sobre la programación del entrenamiento de fuerza en este grupo de edad.

Se propone realizar de ocho a diez ejercicios que involucren los principales grupos musculares, con una frecuencia de dos veces por semana en días no consecutivos $\mathrm{y}$ con resistencias que permitan hacer de doce a quince repeticiones, y de quince a veinte para mejorar la resistencia muscular. El carácter del esfuerzo, según la escala de percepción subjetiva de 10 puntos, es de moderado (5-6 puntos) a alto (7-8 puntos) (Nelson, et al., 2007) o también, intensidades del $40-50 \%$ de 1RM para mejorar la resistencia muscular, o del $20-50 \%$ de 1 RM para mejorar la potencia (Garber et al., 2011). Se sugieren medios como pesas de distinto kilaje, máquinas de musculación, dispositivos neumáticos, bandas elásticas o subir escaleras (Chodzko-Zajko et al., 2009; Garber, et al., 2011). También los autores dan la opción de un entrenamiento de alta intensidad en adultos mayores con suficiente aptitud y experiencia y que trabajen en entornos supervisados. Sobre la potencia, Tschopp et al. (2011), en un meta-análisis, observaron que la mayoría de los estudios utilizaron sesiones de entrenamiento con 2-3 series de 8-12 repeticiones tres veces por semana durante un período de 8-16 semanas, con un máximo de 24 semanas, todo lo anterior a una alta velocidad y baja carga que estaba entre $40 \%-75 \%$ de 1 RM.

En una revisión de Marques et al. (2013) sobre seis bases de datos abarcando estudios originales con entrenamientos para mejorar la fuerza muscular y la potencia en personas mayores y publicados entre 1982 y 2012, se discutió que las recomendaciones del ACSM están dadas a partir de datos que incluyen sujetos de distintas condiciones clínicas y se aplican distintos protocolos de evaluación, por lo que la intensidad calculada desde parámetros diversos incrementa la discrepancia entre los estudios y la reproductibilidad de los datos. Los autores indican que el porcentaje de fuerza donde se alcanza mayor velocidad es aproximadamente al 30\% de la fuerza isométrica pico y entre el $30 \%-50 \%$ de $1 \mathrm{RM}$ y por lo que, para maximizar la potencia mecánica, recomiendan una carga entre $40 \%-60 \%$ y $60 \%-75 \%$ de1RM, para extremidades superiores e inferiores respectivamente, dado que la carga externa necesita ser específica para el tipo de entrenamiento. Por último, discuten sobre el desentrenamiento, tema muy importante por las dinámicas normales de las personas mayores cuando participan de 
programas de ejercicios (viajes, incapacidades, asuntos familiares, etc.), señalando que poco se sabe de sus efectos en esta población, y menos frente a condiciones clínicas específicas.

Otra opción de entrenamiento de fuerza en personas mayores es el ejercicio excéntrico, que ha sido profusamente investigado en sus aplicaciones en el contexto de la salud. Sobre esto, Roig et al. (2008) hicieron una revisión sistemática que incluyó estudios entre 1966 y 2006, donde participaron pacientes con diferentes tipos de enfermedad y se buscaba determinar la eficacia y adecuación del ejercicio excéntrico para restaurar la función musculoesquelética y saber si hay características comunes y específicas de la enfermedad que lo determinen. Se revisaron nueve bases de datos con programas de mínimo cuatro semanas, frecuencia dos días semanales y dos series de diez repeticiones para fuerza, o veinte minutos para resistencia, los pacientes con al menos 18 años y no se limitó el tipo de estudios. Se utilizó la escala PEDro (The Physiotherapy Evidence Database). Se concluyó que el entrenamiento excéntrico puede usarse con seguridad para restaurar la función musculoesquelética en pacientes con algunas enfermedades crónicas específicas, no se halló consenso sobre un programa tipo y se advirtió sobre la dificultad de extrapolar resultados y recomendaciones clínicas para la estandarización de este ejercicio, señalándose la necesidad de hacer mucha más investigación al respecto.

Por otra parte, aunque muchas de las condiciones crónicas de salud antes mencionadas que se favorecen con el entrenamiento excéntrico se presentan en personas mayores, la relación con el envejecimiento también ha sido estudiada. LaStayo et al. (2003) determinaron si un entrenamiento excéntrico de once semanas tres veces por semana entre 10-20 min por sesión podría limitar o incluso revertir la sarcopenia, la discapacidad y las limitaciones funcionales relacionadas en ancianos frágiles. Se lograron ganancias en fuerza sin efectos adversos como dolor muscular o alteraciones miofibrilares y se hallaron cambios en CSA de las fibras en el vasto externo y en general se redujo de mayor a menor el riesgo de caídas. Con esto, se consideró que el ejercicio excéntrico puede ser una importante medida para compensar las alteraciones musculares y revertir significativamente la pérdida de independencia funcional en este grupo.
Una de las muchas explicaciones a las posibilidades del ejercicio excéntrico en personas mayores está relacionada a la preservación de este componente de la fuerza (excéntrica) aun en esta etapa de la vida, y sobre esto, Roig et al. (2010) realizaron otra revisión sistemática en la que concluyen que la evidencia sugiere un conjunto de tres posibles mecanismos que condicionan dicha preservación, por la interconexión de componentes neurológicos, mecánicos y celulares. Primero, relacionados con la disminución de la activación agonista o incremento de la antagonista durante la acción concéntrica; segundo, el incremento del tejido conectivo y la rigidez muscular pasiva (stiffness) atribuida a la edad y tercero, la preservación, aun en la vejez, de la tensión durante la elongación de las fibras musculares.

\section{EFECTOS DEL ENTRENAMIENTO DE LA FUERZA EN PERSONAS MAYORES}

En tiempo reciente, este tema ha sido investigado mediante revisiones sistemáticas y meta-análisis, de los cuales algunos se reseñan a continuación. Latham et al. (2004) realizaron una revisión sistemática sobre el entrenamiento progresivo de la fuerza usado en rehabilitación de personas mayores; incluyeron 62 ensayos clínicos con grupo control y un total de 3.672 sujetos que tenían diferente condición clínica, también hubo variabilidad de intervenciones. Se encontró que este tipo de entrenamiento tiene un gran efecto sobre la fuerza, pero este es pequeño o moderado sobre algunas limitaciones funcionales y la evidencia no es suficiente para indicar que se mejoran los niveles de discapacidad física. En esto último, sugieren que quizás deba combinarse con otras formas de entrenamiento (ej.: equilibrio) y prestar atención a otros factores que contribuyen a la discapacidad, como la autoeficacia, la motivación y la adherencia. Los datos no permitieron una evaluación adecuada de los riesgos asociados a este tipo de entrenamiento, aunque en muchas intervenciones eran evidentes algunos eventos adversos, principalmente musculoesqueléticos. Por último, los autores resaltaron la baja calidad metodológica de los estudios por ausencia de características de diseño que aumentaran la validez interna de sus resultados (ej.: aleatorización, doble ciego) y recomiendan cautela al concluir sobre estos resultados, pues los efectos del entrenamiento progresivo podrían estar sobreestimados. 
Por su parte, Steib et al. (2010) hicieron un meta-análisis de estudios que comparaban múltiples grupos de personas mayores que recibían distintas dosis de entrenamientos de fuerza. No obstante, la cantidad de estudios fue limitada y los autores relativizaron los resultados a parámetros, músculos y población específica incluida en esos estudios. Se encontró que la evidencia es fuerte respecto a que entrenamientos de alta intensidad (entre el 60\%-80\% de 1-RM) producen mejoras en la fuerza máxima; también que bajas intensidades de entrenamiento posiblemente son suficientes para iniciar mejoras neuromusculares y parece requerirse altas intensidades para lograr hipertrofia. La evidencia es moderada cuando se trata de mejorías en la funcionalidad, aunque ésta se evalúa mediante diversidad de pruebas y los autores explican que no siempre las mejoras de la fuerza son verificables de este modo. La evidencia es conflictiva cuando se mira el efecto de diferentes intensidades sobre la resistencia muscular, pudiendo influir las diferencias entre poblaciones estudiadas y el volumen total del entrenamiento.

Sobre el tipo de entrenamiento, los autores (Steib et al., 2010) mencionan que la potencia muestra alta correlación con la capacidad funcional (CF), más de lo que ocurre con la fuerza. Por tanto, un entrenamiento de este tipo es más efectivo que el entrenamiento progresivo de la fuerza para mejorar la potencia, que optimizaría la CF, aunque aclaran que el nivel de evidencia es moderado. Llama la atención que, respecto al entrenamiento excéntrico, mencionan que la evidencia es conflictiva, por cuanto los métodos de sobrecarga excéntrica difieren (trabajo negativo dinámico, versus isocinético).

Sobre el entrenamiento funcional (Functional-Task Resistance Trainning) Steib et al. (2010) mencionan que favorece tareas diarias como subir escaleras o la velocidad de caminar, aunque no hay suficiente información sobre cómo se da en otras tareas cotidianas. No parece haber diferencia entre entrenamiento de pesas versus el Functional-Task y concluyen que este es positivo para las AVD. Por otra parte, se indica que la evidencia es limitada en relación con la frecuencia de entrenamiento y no hay claridad sobre mejores efectos si se hace una, dos o tres veces semanales. Se necesitan más investigaciones respecto a la dosis más adecuada y el tipo de entrenamiento de fuerza para mejorar el rendimiento físico de los adultos mayores.
Dos recientes meta-análisis de Peterson et al. (2010 y 2011) sobre efectos del entrenamiento de fuerza en personas mayores, hechos con la metodología PRISMA (Preferred Reporting Items for Systematic Reviews and Meta-Analyses) indagaron el efecto de dicho entrenamiento sobre la manifestación de la fuerza en miembros superiores e inferiores (Peterson et al., 2010) y sobre la masa libre de grasa (MLG) (Peterson et al., 2011). En el primero, tuvieron por objetivo examinar los efectos de este ejercicio ejecutado en distintos grupos musculares (miembros superiores e inferiores) a través de múltiples regímenes de dosificación y sus resultados sobre la fuerza. Se incluyeron 47 estudios que cumplían los criterios y en total reunieron 1.079 sujetos de ambos sexos con rango de edad entre 50 y 92 años (edad promedio $67,4 \pm 6,3$ años). Se observó una fuerte asociación entre el entrenamiento de fuerza y mejorías significativas de esta capacidad en miembros superiores y aun mayor en los inferiores. Se observaron ganancias promedio de $29 \%$ en la prensa de piernas, $33 \%$ en la extensión de la rodilla, $24 \%$ en el press de pecho y $25 \%$ en tirón de polea o latpull, lo que confirma el valor que esto tiene para la prevención o tratamiento de las disminuciones en la función muscular relacionadas con la edad y para la prevención de la discapacidad.

En el segundo meta-análisis, los autores (Peterson et al., 2011) indican que hasta esa fecha los efectos del entrenamiento de fuerza sobre la MLG eran tratados de modo secundario en los estudios y los pocos metaanálisis referidos al tema resultaban contradictorios, por lo que se propusieron determinar estos efectos sobre la MLG en personas mayores de ambos sexos. En el resultado final se incluyeron 49 estudios que eran ensayos clínicos o cuasiexperimentos en personas mayores de 50 años (1.328 en total) con edades entre 50-83 años (edad promedio 65,5 $\pm 6,5$ ) y donde se midiera la MLG bien fuera por hidrodensitometría, pletismografía o DXA (Absorciometría Dual por Rayos X). Los autores concluyeron que hay una asociación sólida entre el entrenamiento de fuerza para todo el cuerpo y el incremento de MLG, de modo que después de una media de 20,5 semanas, en ambos sexos se presenta un efecto significativo equivalente a aumentos de $1,1 \mathrm{~kg}$ de MLG; sin embargo, no fue posible referirse a otros cambios conexos, como la CSA de la fibra, el volumen muscular, diferenciación en la concentración de células 
satélite, o cambios en el angulopenación o la longitud sarcómero, aspectos morfológicos fundamentales asociados a la CF.

Respecto al entrenamiento de la potencia, Tschopp, et al. (2011) hicieron un meta-análisis comparando los efectos de entrenamientos de fuerza a baja velocidad versus los que incluían velocidades más altas y donde participaron personas mayores. Incluyeron ensayos aleatorios o ensayos con asignaciones diferentes clasificando 11 estudios que vincularon a 377 casos. Encontraron un efecto entre pequeño y mediano sobre las respuestas funcionales a favor del entrenamiento de potencia al compararse con el de fuerza convencional, aunque con un intervalo de confianza muy amplio, lo cual podría hacerlo clínicamente irrelevante. Sobre la seguridad de este entrenamiento no pudieron concluir por el bajo número de participantes. No obstante, parece existir consenso entre distintos autores (Steib et al., 2010; Liu y Latham, 2011; Marques et al., 2013) sobre la indiscutible asociación entre potencia y CF, así como las posibilidades de transferir estos efectos en la reducción del riesgo de discapacidad física en personas mayores.

\section{ASOCIACIONES ENTRE FUERZA Y CAPACIDAD FUNCIONAL}

Como se mencionó anteriormente, la disminución de la masa muscular y los cambios en la funcionalidad de este tejido como características del proceso de envejecimiento afectan la fuerza y sus distintas manifestaciones en las personas mayores, reflejándose en su CF. Las disminuciones en la fuerza, especialmente para las acciones concéntricas, afectan en mayor proporción a los miembros inferiores y se dan a un ritmo más acelerado para el caso de la potencia; estos déficits son predictores de discapacidad y de mayor riesgo de mortalidad en personas mayores (Chodzko-Zajko et al., 2009).

Por lo anterior, el objetivo de entrenar la fuerza en esta población apunta a aumentar o mantener la masa y la funcionalidad del tejido muscular y que en todo caso se refleje del mismo modo en la CF. Este concepto, según Rikli y Jones (2012), alude a la habilidad física para realizar las tareas de la vida diaria, de modo seguro e independiente y sin fatiga excesiva. La relación entre fuerza y CF en este grupo etario se ha asociado al compromiso en las tareas motoras como levantarse y sen- tarse de una silla, la marcha, la agilidad y el equilibrio dinámico, entre otros (Chodzko-Zajko et al., 2009). Esta asociación se ha reconocido desde lo hallado en estudios correlacionales hasta los efectos comprobados en diseños experimentales. En el primero de los casos, se ha establecido que hay relación entre la fuerza evaluada isocinéticamente y la velocidad de la marcha (Ostchega et al., 2004; García et al., 2011) у a su vez la fuerza es un importante predictor del tiempo de caminata en ambos sexos, después de ajustar por edad, raza/etnia, peso y altura (Ostchega et al., 2004). También, bajos niveles de fuerza medidos por prensión manual, baja velocidad de la marcha y baja fuerza en extensores de rodilla se relacionan con un alto riesgo de discapacidad (Guralnik et al., 1995; Vermeulen et al., 2011). Además, son bien conocidas las asociaciones entre el tiempo para levantarse de una silla con la potencia y la fuerza isométrica de extensores de rodilla cuando estas se hacen relativas al peso corporal (Skelton et al., 1994).

En general, desde hace casi un par de décadas, varios estudios encontraron que en las personas mayores la CF mejora por las ganancias producto del entrenamiento de fuerza (Fiaratone et al., 1990; Skelton et al., 1994 y 1995), y eso se ha ido enfocando especialmente en las alcanzadas con los programas orientados a mejorar la potencia (Macaluso y De Vito, 2004; Bottaro et al., 2007). En tal sentido, se ha documentado una mejor transferencia de los efectos de este entrenamiento a esta capacidad, cuando el mismo se ejecuta con alta velocidad y baja sobrecarga. (Marques et al., 2013; Sayers, 2008). Sobre esto, Sayers y Gibson (2010) dicen que ejercitarse de este modo puede imitar aquellos movimientos funcionales más que otras formas de entrenamiento a mayores porcentajes de 1RM y por ende transferir las ganancias de dicho entrenamiento de mejor manera a las actividades que se ven representadas en este tipo de acciones: subir escaleras, levantarse de una silla, restablecer el equilibrio, acelerar súbitamente la marcha ante un imprevisto, entre otros. Cada vez más estudios refuerzan la tesis que la mejora de la CF en personas mayores se da a partir de los trabajos de fuerza que involucren alta velocidad de ejecución. 


\section{CONCLUSIÓN}

De la revisión de la bibliografía podemos concluir que en los últimos 25 años ha cambiado la consideración sobre la importancia que la fuerza tiene en el mantenimiento de la capacidad funcional y autonomía de las personas mayores, ante la evidencia epidemiológica de la relación entre el nivel de fuerza que presentan las personas mayores y la morbilidad y mortalidad de estas.

En estos 25 años ha habido tiempo para que se acuñara el concepto de sarcopenia y que los criterios diagnósticos fueran modificados en 2010, no siendo actualmente suficiente la pérdida de masa muscular sino que esta ha de acompañarse de merma de la fuerza o la performance.

También se ha reconocido con base en los estudios científicos que el papel que el musculoesquelético realiza trasciende su implicación en el mantenimiento

\section{REFERENCIAS BIBLIOGRÁFICAS}

Barbat-Artigas, S., Rolland, Y., Zamboni, M. y AubertinLeheudre, M. (2012). How to assess functional status: A new muscle quality index. The Journal of Nutrition, Health \& Aging, 16(1), 67-77.

Bottaro, M., Machado, S. N., Nogueira, W., Scales, R. y Veloso, J. (2007). Effect of high versus low-velocity resistance training on muscular fitness and functional performance in older men. European Journal of Applied Physiology, 99(3), 257-264.

Brandt, C. y Pedersen, B. K. (2010). The role of exerciseinduced myokines in muscle homeostasis and the defense against chronic diseases. BioMed Research International, 2010Epub Mar 9.

Chodzko-Zajko, W., Proctor, D.N., Fiatarone, M.A., Minson, C.T., Nigg, C.R., Salem, G.J., Skinner, J.S. (2009). Exercise and physical activity for older adults. Medicine and Science in Sports and Exercise, 41(7), 1510-1530.

Cruz-Jentoft, A. J., Baeyens, J. P., Bauer, J. M., Boirie, Y., Cederholm, T., Landi, F. y Schneider, S. M. (2010). Sarcopenia: European consensus on definition and diagnosis report of the European Working Group on Sarcopenia in Older People. Age and Ageing, 39(4), 412-423.

Deschenes, M. R. (2004). Effects of aging on muscle fibre type and size. Sports Medicine, 34(12), 809-824.

Febbraio, M. y Pedersen, B. K. (2005). Contraction-induced myokine production and release: Is skeletal muscle an endocrine organ? Exercise and Sport Sciences Reviews, 33(3), 114-119. postural o el movimiento, siendo importante también en la homeostasis energética y como órgano de liberación de sustancias proteicas que ejercen su efecto sobre tejidos o células que se encuentran a distancia.

Igualmente, se ha ido cambiando el paradigma de ejercicio recomendado en personas mayores, siendo actualmente el entrenamiento de fuerza también aconsejado, y a pesar de la evidencia mostrada por los meta-análisis de la eficacia del entrenamiento de la fuerza funcional y de la existencia de guías para el entrenamiento de fuerza en mayores se precisan más investigaciones sobre el modo, la frecuencia y la intensidad óptimas en personas mayores.

Fiatarone, M. A., Marks, E., Ryan, N. D., Meredith, C. N., Lipsitz, L. y Evans, W. J. (1990). High-intensity strength training in nonagenarians. JAMA, 263(22), 3029-3034.

FitzGerald, S. J., Barlow, C. E., Kampert, J. B., Morrow, J. R., Jackson, A. W., \& y Blair, S. N. (2004). Muscular fitness and all-cause mortality: prospective observations. Journal of Physical Activity \& Health, 1, 7-18.

Frontera, W. R., Hughes, V. A., Fielding, R. A., Fiatarone, M. A., Evans, W. y Roubenoff, R. (2000). Aging of skeletal muscle: A 12-yr longitudinal study. Journal of Applied Physiology, 88(4), 1321-1326.

Frontera, W. R., Meredith, C. N., O'reilly, K. P., Knuttgen, H. G., y Evans, W. J. (1988). Strength conditioning in older men: skeletal muscle hypertrophy and improved function. Journal of Applied Physiology, 64(3), 10381044.

Garber, C. E., Blissmer, B., Deschenes, M. R., Franklin, B. A., Lamonte, M. J., Lee, I. M., ... y Swain, D. P. (2011). American College of Sports Medicine position stand. Quantity and quality of exercise for developing and maintaining cardiorespiratory, musculoskeletal, and neuromotor fitness in apparently healthy adults: guidance for prescribing exercise. Medicine and Science in Sports and Exercise, 43(7), 1334-1359.

García, P. A., Días, J., Días, R. C., Santos, P. y Zampa, C. C. (2011). A study on the relationship between muscle function, functional mobility and level of physical activity in community-dwelling elderly. Revista Brasileira De Fisioterapia, 15(1), 15-22. 
Guralnik, J. M., Ferrucci, L., Simonsick, E. M., Salive, M. y Wallace, R. B. (1995). Lower-extremity function in persons over the age of 70 years as a predictor of subsequent disability. New England Journal of Medicine, 332(9), 556-562.

Janssen, I., Heymsfield, S. B., y Ross, R. (2002). Low relative skeletal muscle mass (sarcopenia) in older persons is associated with functional impairment and physical disability. Journal of the American Geriatrics Society, 50(5), 889-896.

Janssen, I., Heymsfield, S. B., Wang, Z. y Ross, R. (2000). Skeletal muscle mass and distribution in 468 men and women aged 18-88 yr. Journal of Applied Physiology, 89(1), 81-88.

Kraemer, W. J., Adams, K., Cafarelli, E., Dudley, G. A., Dooly, C., Feigenbaum, M. S., ... . y Hoffman, J. R. (2002). American College of Sports Medicine Position Stand. Progression models in resistance training for healthy adults. Medicine and Science in Sports and Exercise, 34(2), 364-380.

LaStayo, P. C., Ewy, G. A., Pierotti, D. D., Johns, R. K., y Lindstedt, S. (2003). The positive effects of negative work: increased muscle strength and decreased fall risk in a frail elderly population. The Journals of Gerontology Series A: Biological Sciences and Medical Sciences, 58(5), M419-M424.

Latham, N. K., Bennett, D. A., Stretton, C. M., y Anderson, C. S. (2004). Systematic review of progressive resistance strength training in older adults. The Journals of Gerontology Series A: Biological Sciences and Medical Sciences, 59(1), M48-M61.

Liu, C. y Latham, N. (2011). Can progressive resistance strength training reduce physical disability in older adults? A meta-analysis study. Disability \& Rehabilitation, 33(2), 87-97.

Macaluso, A. y De Vito, G. (2004). Muscle strength, power and adaptations to resistance training in older people. European Journal of Applied Physiology, 91(4), 450-472.

Marques, M. C., Izquierdo, M. y Pereira, A. (2013). High-speed resistance training in elderly people: A new approach toward counteracting age-related functional capacity loss. Strength \& Conditioning Journal, 35(2), 23-29.

Nelson, M. E., Rejeski, W. J., Blair, S. N., Duncan, P. W., Judge, J. O., King, A.y Castaneda-Sceppa, C. (2007). Physical activity and public health in older adults: Recommendation from the American College of Sports Medicine and the American Heart Association. Medicine and Science in Sports and Exercise, 39(8), $1095-1105$.

Ostchega, Y., Dillon, C. F., Lindle, R., Carroll, M., y Hurley, B. F. (2004). Isokinetic leg muscle strength in older americans and its relationship to a standardized walk test: Data from the national health and nutrition examination survey 1999-2000. Journal of the American Geriatrics Society, 52(6), 977-982.

Pedersen, B. K. y Febbraio, M. A. (2008). Muscle as an endocrine organ: Focus on muscle-derived interleukin-6. Physiological Reviews, 88(4), 13791406.

Pedersen, B. K. (2011). Muscles and their myokines. The Journal of Experimental Biology, 214(2), 337-346.

Pedersen, B., Bruunsgaard, H., Ostrowski, K., Krabbe, K., Hansen, H., Krzywkowski, K.e Ibfelt, T. (2000). Cytokines in aging and exercise. International Journal of Sports Medicine, 21(Sup. 1), 4-9.

Petersen, A. M. W. y Pedersen, B. K. (2005). The antiinflammatory effect of exercise. Journal of Applied Physiology, 98(4), 1154-1162.

Peterson, M. D., Rhea, M. R., Sen, A. y Gordon, P. M. (2010). Resistance exercise for muscular strength in older adults: A meta-analysis. Ageing Research Reviews, 9(3), 226-237.

Peterson, M. D., Sen, A. y Gordon, P. M. (2011). Influence of resistance exercise on lean body mass in aging adults: A meta-analysis. Medicine and Science in Sports and Exercise, 43(2), 249-258.

Raj, I. S., Bird, S. R. y Shield, A. J. (2010). Aging and the force-velocity relationship of muscles. Experimental Gerontology, 45(2), 81-90.

Ratamess, N. A., Alvar, B. A., Evetoch, T. E., Housh, T. J., Ben Kibler, W., Kraemer, W. J. y Triplett, N. T. (2009). Progression models in resistance training for healthy adults. Medicine and Science in Sports and Exercise, 41(3), 687-708.

Rikli, R. E. y Jones, C. J. (2012). Senior fitness test manual. Human Kinetics 186p.

Roig, M., MacIntyre, D. L., Eng, J. J., Narici, M. V., Maganaris, C. N. y Reid, W. D. (2010). Preservation of eccentric strength in older adults: Evidence, mechanisms and implications for training and rehabilitation. Experimental Gerontology, 45(6), 400-409.

Roig, M., Shadgan, B. y Reid, W. D. (2008). Eccentric exercise in patients with chronic health conditions: a systematic review. Physiotherapy Canada, 60(2), 146-160.

Rosenberg, I. H. (1997). Sarcopenia: Origins and clinical relevance.The Journal of Nutrition, 127(5), 990S-991S.

Sayers, S. P. (2008). High velocitypower training in olderadults. CurrAgingSci, 1(1), 62-67.

Sayers, S. P. y Gibson, K. (2010). A comparison of high-speedpower training and traditionalslowspeedresistance training in oldermen and women. The Journal of Strength \& Conditioning Research, 24(12), 3369-3380. 
Skelton, D. A., Greig, C. A., Davies, J. M., \& Young, A. (1994). Strength, power and related functional ability of healthy people aged 65-89 years. Age and Ageing, 23(5), 371-377.

Skelton, D. A., Young, A., Greig, C. A. y Malbut, K. E. (1995). Effects of resistance training on strength, power, and selected functional abilities of women aged 75 and older. Journal of the American Geriatrics Society, 43(10), 1081-1087.

Steib, S., Schoene, D. y Pfeifer, K. (2010). Dose-response relationship of resistance training in older adults: A meta-analysis. Medicine and Science in Sports and Exercise, 42(5), 902-914.

Szulc, P., Duboeuf, F., Marchand, F. y Delmas, P. D. (2004). Hormonal and lifestyle determinants of appendicular skeletal muscle mass in men: The MINOS study. The American Journal of Clinical Nutrition, 80(2), 496-503.
Trappe, T. (2009). Influence of aging and long-term unloading on the structure and function of human skeletal muscle. Applied Physiology, Nutrition, and Metabolism, 34(3), 459-464.

Tschopp, M., Sattelmayer, M. K. y Hilfiker, R. (2011). Is power training or conventional resistance training better for function in elderly persons? A meta-analysis. Age and Ageing, 40(5), 549-556.

Vermeulen, J., Neyens, J. C., Van Rossum, E., Spreeuwenberg, M. D. y De Witte, L. P. (2011). Predicting ADL disability in community-dwelling elderly people using physical frailty indicators: A systematic review. BMC Geriatrics, 11(1), 33. 